\title{
Effects of In-plane Elastic Stress and Normal External Stress on Viscoelastic Thin Film Stability
}

\author{
F. Closa ${ }^{1}$, F. Ziebert ${ }^{2,3}$, E. Raphaël ${ }^{1 *}$ \\ ${ }^{1}$ Laboratoire de Physico-Chimie Théorique - UMR CNRS Gulliver 7083, ESPCI \\ 10 rue Vauquelin, F-75231 Paris, France \\ ${ }^{2}$ Physikalisches Institut, Albert-Ludwigs-Universität, 79104 Freiburg, Germany \\ ${ }^{3}$ Institut Charles Sadron, 23 rue du Loess, 67034 Strasbourg, France
}

\begin{abstract}
Motivated by recent experiments on the electro-hydrodynamic instability of spincast polymer films, we study the undulation instability of a thin viscoelastic polymer film under in-plane stress and in the presence of either a close by contactor or an electric field, both inducing a normal stress on the film surface. We find that the in-plane stress affects both the typical timescale of the instability and the unstable wavelengths. The film stability is also sensitive to the boundary conditions used at the film-substrate interface. We have considered two conditions, either rigidly attaching the film to the substrate or allowing for slip.
\end{abstract}

Keywords and phrases: thin films, polymers, instability, mechanics, (visco)elasticity, internal/residual stress

Mathematics Subject Classification: 74B20, 74D10, 74F15, 74H55, 74K35, 76E17

\section{Introduction}

Polymeric thin films are widely used in technological applications. In the liquid state they are used, for example, as lubricants while in the solid, most often glassy, state they can protect surfaces or give them a desired property. Clearly the stability of such coatings is a crucial issue determining their applicability and reliability.

Thin polymer films are usually produced starting from a dilute solution by various coating techniques. A very popular method is spin-coating [15], as it results in very thin (down to tens of nanometers) and homogeneous films without pronounced surface roughness. However, spin-coating is a rather harsh process: the solvent usually evaporates on time scales much faster than the typical time the increasingly concentrated polymer solution (or finally the melt) needs to establish equilibrium conformations and equilibrium bulk-like properties. This is even more the case when the film thickness reaches some tens of nanometers and becomes of the order of, or even smaller, than the equilibrium coil size of the polymers. Hence spin-coated films are generally believed to be stressed, mostly in the plane of the film, as evidenced e.g. by dewetting experiments $[4,18]$.

${ }^{*}$ Corresponding author. E-mail: elie.raphael@espci.fr 


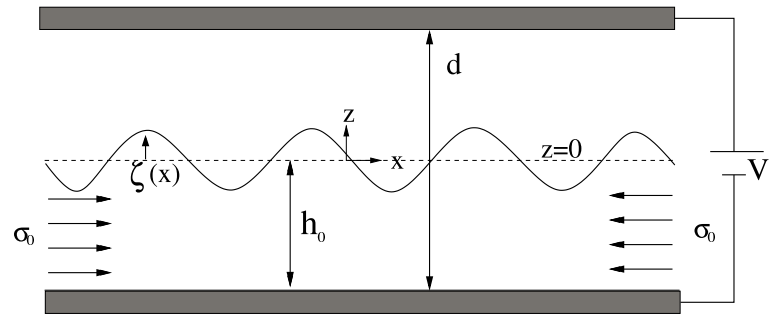

Figure 1. Sketch of the capacitor geometry. The thin polymer film is supposed to be under uniaxial stress $\sigma_{0}$ in $x$-direction (which could also be tensile). An external electric field in $z$-direction (for the unperturbed film) is imposed by externally applying a voltage $V$ between two electrodes a distance $d$ away. The gap can be filled with any dielectric having a differing dielectric constant from the polymer film.

Unfortunately, so far one does not have a good understanding of, and hence no handle on, these stresses. Valuable insight can however be extracted by the study (and careful comparison to the non-stressed case) of the various instabilities of thin polymer films reported recently:

(i) As already mentioned, if a very thin film is forced to cover a surface that it does not like, fluidizing the film by heating beyond the glass transition temperature induces dewetting of the film. From a rheological analysis [4] or the dynamics of the evolving rim [18] one can extract information about an averaged stress. Unfortunately, as the rim evolution is due to a complex interplay of the residual stress, the viscoelasticity and the dissipation/friction at the substrate $[24,27]$, it may be hard to deconvolute these processes from the (time-dependent since relaxing) stress when interpreting experimental data.

(ii) A second well-known instability is the finger-like instability of the contact/adhesion front of a thin polymer layer sandwiched between the substrate and a thin elastic confining sheet (the geometry being similar to the peel test of an adhesive tape). This instability so far has been studied [9] only for rather thick films (micrometers) not prone to internal stresses due to the preparation process and has been described theoretically by various approaches $[1,7,25]$. The effect of external in-plane stress in this geometry has been studied [8] and shown to transform the sinusoidal-like fingers into triangular shapes. It might be difficult but possible to down-scale the film thickness to the range (below $100 \mathrm{~nm}$ ) where internal stress should arise and to study the shape of the fingers as an indicator of stress.

(iii) One of the most promising setup, that will also be studied in this work, makes use of the socalled electro-hydrodynamic instability [19], see the sketch in Fig. 1. There the polymeric film is brought between the two plates of a capacitor and an electric field is applied normal to the film. Due to the dielectric mismatch between the film and the gap (usually either filled with air or a liquid), a Maxwell stress arises that drives the dielectric interface in a position of less energy, i. e. parallel to the field lines [23]. In the early stage of this instability, the surface starts to undulate with a characteristic wavelength. The growth rates of the undulations have been recently obtained by interferometry [3] and freshly coated (presumably stressed) films could be compared to aged ones (un- or less stressed).

In the absence of stress, the electro-hydrodynamic instability has been studied to a large extent for simple liquids [13,21,23], but less for viscoelastic materials (solid or liquid) [20,22]. If one wants to include stress for the viscoelastic case, one has to be aware of another instability known for elastic solids as the so-called Asaro-Tiller-Grinfeld mechanism $[2,10]$. This instability describes the fact that the system can decrease its elastic energy, if a material transport mechanism is allowed, by undulating the surface and thereby relaxing stress. In purely elastic systems, material transport can originate from either crystallization/melting in the case of crystal films in contact with its melt, or surface diffusion. We recently studied this effect for an elastic polymer film including surface diffusion [6]. In the viscoelastic case studied in this paper, the film has liquid-like mobility and the (slow) diffusion is an additional 
contribution to the material transport. For simplicity, diffusion mechanism will be neglected in the present work.

The present work is organized as follows: in section 2 we describe a nonlinear formulation for the problem of a stressed viscoelastic film and discuss two different generic boundary conditions between the film and the substrate: slip and fixed. In section 3, we perform a general linear analysis of the stressed state with respect to surface undulations, followed by a detailed analysis and discussion of the two boundary conditions in section 4 . In view of the fact that the instabilities (ii) and (iii) discussed above share similarities with the instability due to van der Waals forces upon approaching a contactor to the free surface of the film (studied e.g. in [20]), we will also discuss this slightly simpler geometry in some detail. Finally, we conclude in section 5 .

\section{Model}

As the detailed structure and the nature of the stresses for a spin-coated film are not known to date, we will apply a simplified but well-defined protocol. Note that to properly describe finite stresses in a thin polymer film, one has to use a nonlinear elasticity formulation.

Nonlinear elasticity formulation: Let us assume that the film was originally in a stress-free state (described by coordinates $\mathbf{X}=X_{i} \mathbf{e}_{\mathbf{i}}$ ). Then we stretch (or compress) the film, for simplicity uniaxially in the $x$-direction by a factor $\lambda>1\left(\lambda<1\right.$, respectively). This state described by coordinates $\mathbf{x}=x_{i} \mathbf{e}_{\mathbf{i}}$ is considered as the base state and is under uniaxial stress $\sigma_{x x}^{0}$, see below. Finally, the film is brought in close contact with the substrate (either still permitting for slip, or perfectly fixed to it, see the discussion of the boundary conditions) and can evolve under the in-plane stress and any additional force acting on it, here the electric Maxwell stress. This current state is described by coordinates $\tilde{\mathbf{x}}=\tilde{x}_{i} \mathbf{e}_{\mathbf{i}}$. For simplicity (and as a good approximation for elastomers and polymer melts) we assume the film to be incompressible.

The total deformation gradient tensor from $\mathbf{X}$ to $\tilde{\mathbf{x}}$ reads

$$
\mathbf{F}=\frac{\partial \tilde{\mathbf{x}}}{\partial \mathbf{X}}=\frac{\partial \tilde{\mathbf{x}}}{\partial \mathbf{x}} \cdot \frac{\partial \mathbf{x}}{\partial \mathbf{X}}=: \mathbf{F}_{\mathbf{2}} \cdot \mathbf{F}_{\mathbf{1}} .
$$

Here

$$
\mathbf{F}_{\mathbf{1}}=\operatorname{Diag}\left(\lambda, \lambda^{-1 / 2}, \lambda^{-1 / 2}\right)
$$

describes the stretching (or compression) of the incompressible film, where Diag specifies the diagonal entries of a $3 \times 3$ diagonal matrix. Note that this step must be formulated in the nonlinear regime, as stresses are finite (and not infinitesimal). Using $\tilde{\mathbf{x}}=\mathbf{x}+\mathbf{U}$, the second tensor,

$$
\mathbf{F}_{\mathbf{2}}=\mathbf{I}+\nabla \mathbf{U}
$$

(with $\mathbf{I}$ the identity tensor) introduces the usual linear displacement gradient tensor $\nabla \mathbf{U}=\left(\partial_{j} U_{i}\right)_{i j}$. It denotes the displacement in the current state with respect to the stretched state (which we refer to as the base state). As we are only interested in the stability of the base state, in this step a linearized theory is enough for our purposes.

Assuming a Neo-Hookean elastic solid [14], the Cauchy stress tensor is defined as

$$
\sigma=G \mathbf{B}-P \mathbf{I},
$$

where $\mathbf{B}=\mathbf{F} \cdot \mathbf{F}^{\mathrm{T}}$ is the left Cauchy-Green tensor. It describes the stress after a deformation in the current configuration per unit of area of the current configuration. $G$ is the shear modulus and $P$ is a Lagrangian multiplier (an effective pressure) that ensures the incompressibility condition.

In the base state, from Eq. (2.4) one directly gets $\sigma_{i j}^{0}=0$ except for

$$
\sigma_{x x}^{0}=G\left(\lambda^{2}-\lambda^{-1}\right)
$$


This establishes a connection between the stretch factor $\lambda$ of our protocol and the prestress $\sigma_{0}=\sigma_{x x}^{0}$ in the film.

Viscoelasticity: There are two basic models of viscoelasticity: the Maxwell model describes the simplest viscoelastic liquid and the Voigt-Kelvin model the simplest viscoelastic solid. In this work we will apply the Voigt-Kelvin model which takes into account viscous flow at short time scales and (non-linear Neo-Hookean) elasticity at long time scales. Thereby we can study the additional effects of transient viscous flows inside the film on the film instability under in-plane stress and normal electric stresses. In the geometry under consideration, the Voigt-Kelvin model is conceptually simpler than the Maxwell model which requires a separate treatment and will be discussed elsewhere.

In the Voigt-Kelvin model, the viscous and elastic degrees of freedom are 'in parallel', hence the stresses add and we can write the Cauchy stress tensor as a sum of elastic and viscous parts, generalizing Eq. (2.4) to

$$
\sigma=\sigma^{E}+\sigma^{V}=G \mathbf{B}+2 \eta \mathbf{D}-P \mathbf{I},
$$

where $\eta$ is the shear viscosity, $\mathbf{D}=\frac{\mathbf{L}+\mathbf{L}^{T}}{2}$ is the symmetric part of the velocity gradient $\mathbf{L}=\nabla \mathbf{V}$ and $\mathbf{V}=\dot{\mathbf{U}}[14,17]$.

Boundary conditions at the substrate: For thinner and thinner films, interfaces become increasingly important because of the increasing surface per volume ratio. This is equally true for the boundary conditions (BCs). Hence we discuss two distinct cases for the $\mathrm{BC}$ of the film at the substrate, to test the sensibility of the results on the BCs.

First of all, at the bottom surface defined by $z=-h_{0}$, where $h_{0}$ is the stretched film thickness (cf. Fig. 1), we impose vanishing normal displacement

$$
U_{z}=0 \quad \text { at } z=-h_{0} .
$$

This simply states that the film is not allowed to detach from the substrate, i.e. we do not consider situations involving delamination. As the second BC needed to close the system, we impose: either the so-called slip BC

$$
\text { slip BC : } \quad \sigma_{x z}=0 \text { at } z=-h_{0},
$$

which allows a displacement along the substrate, but no shear stress. Experimentally, this can be obtained by spin-coating a polymer film on a incompatible polymer brush attached to the substrate (as used e.g. in [18]). In contrast, the so-called fixed BC

$$
\text { fixed } \mathrm{BC}: \quad U_{x}=0 \text { at } z=-h_{0}
$$

describes the case where the film is strongly attached (e.g. by chemical bonds) to the substrate.

Boundary conditions at the free surface: At the free surface defined as $z=\zeta(x)$, cf. Fig. 1 , and assuming a quasi-static evolution, the normal-normal component of stress has to balance the surface tension whereas the shear stress has to vanish. The surface tension $\gamma$ is acting against surface modulations. In addition to the elastic stress, assuming a perfect dielectric polymer, the electric field induces an electric contribution described by the Maxwell tensor [11]

$$
T_{i j}^{(\alpha)}=\epsilon_{\alpha} \epsilon_{0}\left(E_{i}^{(\alpha)} E_{j}^{(\alpha)}-\frac{1}{2}\left(E^{(\alpha)}\right)^{2} \delta_{i j}\right) .
$$

Here the index $\alpha=1$ denotes the polymer film and $\alpha=2$ the gap ( $\epsilon_{2}=1$ for air). Denoting the unit vectors normal and tangential to the surface by $\hat{\mathbf{n}}$ and $\hat{\mathbf{t}}$, respectively, the normal stress BC at the free surface reads to leading order

$$
\hat{\mathbf{n}} \cdot\left(\sigma^{(1)}-\sigma^{(2)}+T^{(1)}-T^{(2)}\right) \cdot \hat{\mathbf{n}}=\gamma \partial_{x}^{2} \zeta .
$$


As one has $\hat{\mathbf{n}} \cdot\left(T^{(1)}-T^{(2)}\right) \cdot \hat{\mathbf{t}}=0$, the tangential BC at the free surface is simply given by

$$
\hat{\mathbf{n}} \cdot\left(\sigma^{(1)}-\sigma^{(2)}\right) \cdot \hat{\mathbf{t}}=0,
$$

not influenced by the electric field.

The electric field has to fulfill the usual electrostatics equations with standard BCs at the dielectric interface [11]. We parameterize the free interface of the film by

$$
z=\zeta(x, t)=\varepsilon h e^{i k x+s t},
$$

where $k=\frac{2 \pi}{l_{w}}$ is the wavenumber (and $l_{w}$ the wavelength) and $s$ the growth rate of the perturbation. Assuming a small surface undulation ( $\varepsilon$ being a book-keeping parameter), Eqs. $(2.11,2.12)$ can be written in compact form as

$$
\sum_{j=1}^{3} \sigma_{i j} n_{j}=\left(F+Y(k) \zeta(x, t)-\gamma k^{2} \zeta(x, t)\right) n_{i}
$$

at first order [where the normal vector reads $\left.\mathbf{n}=\left(-\partial_{x} \zeta, 0,1\right)\right]$. The first two terms on the r.h.s. are the electric (Maxwell stress) contributions. Clearly, these contributions are independent of the viscoelastic properties and have been already calculated previously $[6,16]$

$$
\begin{aligned}
F & =\frac{1}{2} \frac{\epsilon_{0} \epsilon_{1} \epsilon_{2}\left(\epsilon_{1}-\epsilon_{2}\right) V^{2}}{\left(\epsilon_{2} h_{0}+\left(d-h_{0}\right) \epsilon_{1}\right)^{2}}, \\
Y(k) & =\frac{2 k F\left(\epsilon_{1}-\epsilon_{2}\right)}{\left[\epsilon_{1} \tanh \left(\left(d-h_{0}\right) k\right)+\epsilon_{2} \tanh \left(h_{0} k\right)\right]} .
\end{aligned}
$$

Note that both $F$ and $Y$ are strictly positive for $\epsilon_{1}>\epsilon_{2}$. The wavelength dependence of $Y(k)$ is due to the boundary conditions for the electric field at the free surface, taking into account that undulations of this surface curve the electric field lines. Often, experiments are carried out with a contactor [20], using van der Waals forces (instead of electric forces in the capacitor geometry) to destabilize the free surface. In this case, we have just to consider $Y(k)$ as a constant: $Y(k)=Y=$ const. with according to [20]

$$
F=\frac{1}{6 \pi} \frac{A}{\left(d-h_{0}\right)^{3}}, \quad Y=\frac{1}{2 \pi} \frac{A}{\left(d-h_{0}\right)^{4}},
$$

where $A$ is the Hamaker constant (typically $A \sim 10^{-19} \mathrm{~J}$ ). Both cases will be discussed in the following.

\section{Analysis}

The bulk viscoelastic problem, neglecting effects of inertia, is given by $\nabla \cdot \sigma=0$. This is the standard elastic force balance [12]. Note, however, that due to viscoelasticity this is a dynamic equation. We will consider a plane strain situation $\left(U_{y}=0\right.$ and none of the variables have y-dependence). Using incompressibility $\partial_{x} U_{x}+\partial_{z} U_{z}=0$, we get

$$
\begin{aligned}
& -\partial_{x} P+G\left(\frac{\partial_{z}^{2} U_{x}}{\lambda}+\lambda^{2} \partial_{x}^{2} U_{x}\right)+\eta\left(\partial_{x}^{2}+\partial_{z}^{2}\right) \dot{U}_{x}=0 \\
& -\partial_{z} P+G\left(\frac{\partial_{z}^{2} U_{z}}{\lambda}+\lambda^{2} \partial_{x}^{2} U_{z}\right)+\eta\left(\partial_{x}^{2}+\partial_{z}^{2}\right) \dot{U}_{z}=0 .
\end{aligned}
$$

Note that in these equations the $x$ - $z$-symmetry is broken by any finite stretch $\lambda \neq 1$ : the base state (stretched state) is anisotropic, as the derivatives in $x$ - and $z$-directions have different prefactors $\left(\lambda^{2}\right.$ vs. $\left.\lambda^{-1}\right)$. 
Eqs. (3.1) have a homogeneous solution, which obviously reads $U_{x}=0=U_{z}$ and, upon imposing the BCs at the free surface, $P=P_{0}=-F+G \lambda^{-1}$, where $F$ is the electric contribution to the pressure given in the last section. To study the stability of this homogeneous base state, the growth rates of small amplitude surface undulations have to be calculated. Using the ansatz

$$
\begin{aligned}
U_{x}(x, z, t) & =\varepsilon u_{x}(z) e^{i k x+s t}, \\
U_{z}(x, z, t) & =\varepsilon u_{z}(z) e^{i k x+s t}, \\
P(x, z, t) & =P_{0}+\varepsilon p_{1}(z) e^{i k x+s t},
\end{aligned}
$$

and incompressibility again, $i k u_{x}+\partial_{z} u_{z}=0$, the equations decouple. The equation for $u_{z}$ reads

$$
l^{2} k^{2} u_{z}-\left(k^{2}+l^{2}\right) u_{z}^{(2)}+u_{z}^{(4)}=0,
$$

where a superscript $(n)$ means the $n$th derivative and

$$
l=k \sqrt{\frac{s \eta \lambda+\lambda^{3} G}{s \eta \lambda+G}}
$$

is an inverse length scale that depends both on the elastic properties (shear modulus and prestrech) and the dynamic properties (viscosity, as well as the time scale of the perturbation). The general solution of Eq. (3.2) for $\lambda \neq 1$ and $G \neq 0$ reads

$$
\begin{aligned}
u_{z}= & A_{1} \cosh \left[k\left(z+h_{0}\right)\right]+A_{2} \cosh \left[l\left(z+h_{0}\right)\right] \\
& +B_{1} \sinh \left[k\left(z+h_{0}\right)\right]+B_{2} \sinh \left[l\left(z+h_{0}\right)\right] .
\end{aligned}
$$

This result is consistent with the pure elastic limit, $\eta=0$, where one regains $l=k \lambda^{3 / 2}[6]$. In the purely viscous case, $G=0$, where $l=k$, the solution of Eq. (3.2) reads

$$
\begin{aligned}
u_{z}= & A_{1} \cosh \left[k\left(z+h_{0}\right)\right]+A_{2}\left(z+h_{0}\right) \cosh \left[k\left(z+h_{0}\right)\right] \\
& +B_{1} \sinh \left[k\left(z+h_{0}\right)\right]+B_{2}\left(z+h_{0}\right) \sinh \left[k\left(z+h_{0}\right)\right],
\end{aligned}
$$

identical to the result obtained in Ref. [22]. Note that for the unstretched, isotropic case one also gets $l=k$ and hence solution (3.5).

In the next section, we will obtain $u_{z}$ for the two different BCs under consideration. A solvability condition then yields an equation for the growth rate of surface undulations $s(k)$. Note that the linear problem is completely solved when $u_{z}$ is obtained, as $u_{x}$ can be obtained from it via incompressibility and $p_{1}$ from either one of Eqs. (3.1).

\section{Fixed vs. slip condition at the substrate}

Fixed BC at the substrate: In the case of the viscoelastic film being rigidly attached to the substrate, the vanishing displacement on the substrate implies

$$
u_{z}\left(-h_{0}\right)=0 \Rightarrow A_{2}=-A_{1}, \quad u_{x}\left(-h_{0}\right)=0 \Rightarrow B_{2}=-\frac{k}{l} B_{1} .
$$

Imposing also the BCs at the free surface, Eq. (2.14), on the general solution (3.4), one gets

$$
\begin{aligned}
& A_{1, f}=h \frac{\left(l^{2}-k^{2}\right)\left[k\left(\lambda^{3}-1\right) G v(k, l, k)+\lambda\left(k^{2} \gamma-Y(k)\right) w(k, l)\right]}{k\left(\lambda^{3}-1\right) G\left[k^{4}+6 k^{2} l^{2}+l^{4}-\left(k^{2}+l^{2}\right) f(k, l)\right]}, \\
& B_{1, f}=-h \frac{\left(l^{2}-k^{2}\right)\left[k\left(\lambda^{3}-1\right) G w(k, l)+\lambda\left(k^{2} \gamma-Y(k)\right) v(k, l, l)\right]}{k\left(\lambda^{3}-1\right) G\left[k^{4}+6 k^{2} l^{2}+l^{4}-\left(k^{2}+l^{2}\right) f(k, l)\right]},
\end{aligned}
$$


where we have introduced the abbreviations

$$
\begin{aligned}
f(k, l) & =(l-k)^{2} \cosh \left[h_{0}(l+k)\right]+(l+k)^{2} \cosh \left[h_{0}(l-k)\right], \\
v(k, l, m) & =\left(l^{2}+k^{2}\right) \cosh \left(h_{0} k\right)-2 m^{2} \cosh \left(h_{0} l\right), \\
w(k, l) & =\left(l^{2}+k^{2}\right) \sinh \left(h_{0} k\right)-2 k l \sinh \left(h_{0} l\right) .
\end{aligned}
$$

The condition for nontrivial, i. e. undulated, solutions to exist is given by

$$
\zeta(x, t)=u_{z}(x, z=0, t)
$$

or $\varepsilon h=u_{z}(0)$. This expresses that for consistency, the displacement at the surface must equal the height perturbation. Alternatively, the system of BCs could have been written as a $4 \times 4$ matrix equation and the roots of the determinant have to be determined.

With $u_{z}$ already calculated, Eq. (4.4) can be written as $h Z(k)=0$ with a wavenumber-dependent function $Z(k)$. If one finds real wavenumbers $k$ with $Z(k)=0$, the film is unstable against periodic undulations; otherwise, $h Z(k)=0$ implies $h=0$ and the film is stable and stays flat. It is suitable to rescale the problem by introducing dimensionless units:

$$
\begin{aligned}
& S=\frac{\eta}{G} s, K=h_{0} k, Y=\frac{h_{0}}{G} Y(k), \quad \Gamma=\frac{\gamma}{G h_{0}} \quad \text { and } \\
& L=L(S, \lambda)=h_{0} l=K \sqrt{\frac{\lambda\left(S+\lambda^{2}\right)}{1+S \lambda}} .
\end{aligned}
$$

In case of the fixed $\mathrm{BC}$ (hence the subscript $f$ ) one gets

$$
\begin{aligned}
Z_{f}= & \left(\Gamma K^{2}-Y\right) \lambda\left(L^{2}-K^{2}\right)^{2}[K \cosh (K) \sinh (L)-L \sinh (K) \cosh (L)] \\
& +K\left(\lambda^{3}-1\right)\left[4 K^{2} L\left(L^{2}+K^{2}\right)+K\left(K^{4}+6 K^{2} L^{2}+L^{4}\right) \sinh (K) \sinh (L)\right. \\
& \left.-L\left(5 K^{4}+2 H^{2} L^{2}+L^{4}\right) \cosh (K) \cosh (L)\right]
\end{aligned}
$$

Upon solving numerically the equation $Z_{f}(S, K, \lambda, Y, \Gamma)=0$, one obtains the growth rate $S_{f}(K)$ of a mode of wave vector $K$ as a function of the prestretch $\lambda$, the renormalized electric stress $Y$ and the renormalized surface tension $\Gamma$.

Fig. 2 displays the growth rates $S_{f}(K)$ for different stretch factors $\lambda$ for typical parameter values of a thin polystyrene film of $\gamma_{P S}=30 \cdot 10^{-3} \mathrm{Nm}^{-1}, \epsilon_{P S}=2.5, G=6 \cdot 10^{5} \mathrm{~Pa}$ and a film thickness of $h_{0}=100 \mathrm{~nm}$. In reduced units this amounts to $\Gamma=0.5$. For the destabilization, we use $d=4 h_{0}$ for the distance of the capacitor electrodes, and an applied voltage of $V=400 \mathrm{~V}$. In case of the contactor, we used $Y=10=$ const.

The unstretched (hence unstressed) case $\lambda=1$ is shown as the black curves in Fig. 2. An analytical formula can be given in this case by taking the limit $\lambda \rightarrow 1$,

$$
S_{f}(K, \lambda=1)=\frac{\left(Y-K^{2} \Gamma\right)(\sinh (2 K)-2 K)-2 K\left(\cosh (2 K)+1+2 K^{2}\right)}{2 K\left[1+2 K^{2}+\cosh (2 K)\right]},
$$

which agrees with results obtained in Ref. [20]: for the contactor case, the critical value $Y_{c}$ corresponding to the lowest van der Waals force to be applied in order for an instability to develop, is given by $Y_{c}=6.22$, and the critical wavenumber by $K_{c}=2.12$, if the surface tension is neglected [20]. Note that in general, $Y_{c}$ for the contactor case, $V_{c}$ for the electric case, and $K_{c}$ are functions of $\Gamma$. The critical values as a function of the stretch factor $\lambda$ are discussed in Figs. 5 and 6 below. 

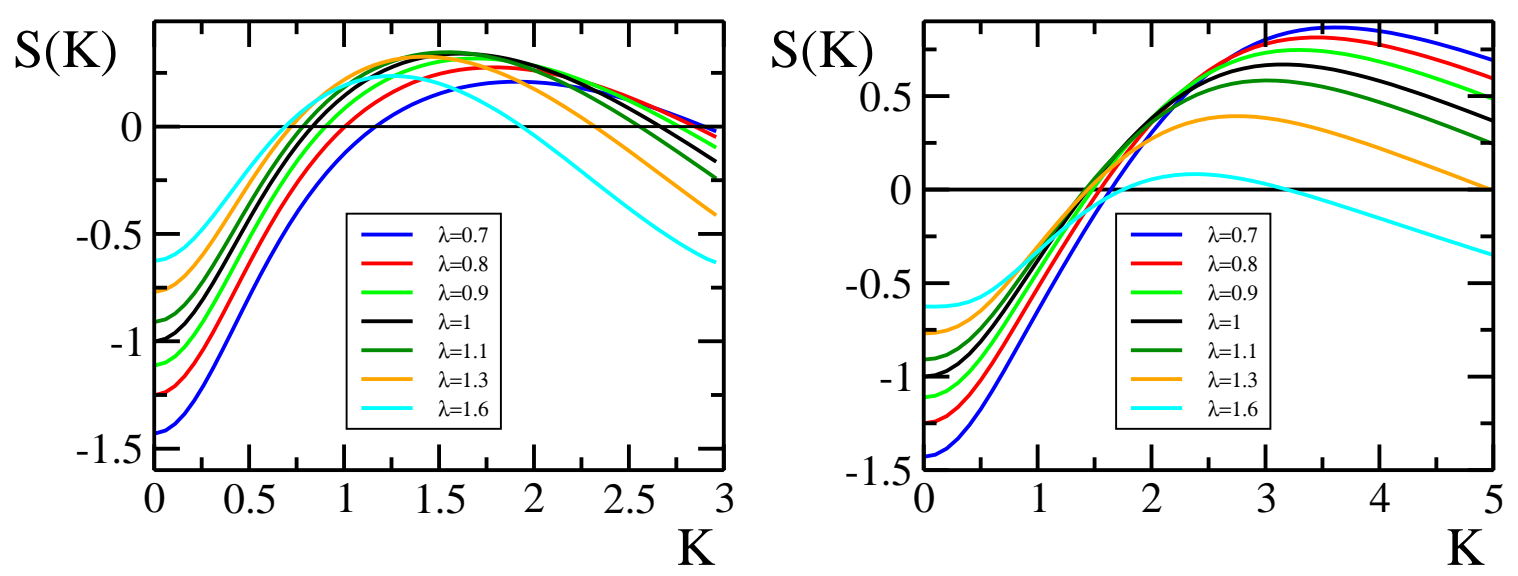

Figure 2. (Color online) The dimensionless growth rates of surface undulations, $S$, as a function of the renormalized wavenumber $K$ for the fixed BC at the bottom. Left panel: case of a contactor $(Y=10=$ const.). With increasing compression $(\lambda<1)$ the wavenumber of the fastest growing mode increases, while it decreases for increasing tension $(\lambda>1)$. Right panel: case of the capacitor geometry (applied voltage $V=400 V$ ). With decreasing stretch factor $\lambda$, the fastest growing wavenumber increases. Both figures account for a typical reduced surface tension of $\Gamma=0.5$.

Fig. 2 shows that for a sufficiently high van der Waals or electric force, wavenumbers within a certain window, namely those with $S(K)>0$, can grow and an instability will develop. The maximum position of $S(K)$ defines $K_{f g}$, the fastest growing mode that dominates the short-time development of the instability and will be observed experimentally. Let us discuss the influence of the stretch factor $\lambda$ and start with the small wavenumbers (large wavelengths). First of all, $K=0$ has to be excluded, as it does not satisfy the incompressibility condition. For the limit $K \rightarrow 0$, one can show $S(K \rightarrow 0)=-1 / \lambda$. Hence if the film is under tension, $\lambda>1$, growth rates are shifted to less negative values while for compression, $\lambda<1$, they are shifted to more negative values. A second noticeable behavior is that tension stabilizes the high wavenumbers as compared to the unstressed case (cf. cyan vs. black curve), while compression destabilizes them (cf. blue vs. black curve). Altogether this leads to the most unstable wavenumber $K_{f g}$ being shifted to smaller wavenumbers in case of tension and higher wavenumbers in case of compression. These observations hold for both destabilization mechanisms: contactor or capacitor. However, Fig. 2 also shows a difference: for the capacitor geometry, the maximum growth rate $S\left(K_{f g}\right)$ always increases when $\lambda$ decreases. Hence compression, $\lambda<1$, is always more destablizing than tension, $\lambda>1$. In contrast, for the contactor case, $S\left(K_{f g}\right)$ does not monotonously increase with a decrease of $\lambda$, but has a maximum for $\lambda \simeq 1.1$.

Slip BC at the substrate: To investigate the sensitivity of the film stability on the BCs at the substrate, we now study the second $\mathrm{BC}$ discussed above. Otherwise the analysis is completely analogous. Allowing for slip at the bottom, the BCs $(2.7,2.8)$ imply

$$
u_{z}\left(-h_{0}\right)=0 \Rightarrow A_{2}=-A_{1}, \sigma_{x z}\left(-h_{0}\right)=0 \Rightarrow A_{2}=0 .
$$

and hence $A_{1, s}=A_{2, s}=0$. Using the BCs (2.14) at the free surface and Eq. (2.16), one gets

$$
\begin{aligned}
B_{1, s} & =-2 h \frac{\left(l^{2}-k^{2}\right)\left[k^{2}\left(\lambda^{3}-1\right) G \cosh \left(h_{0} l\right)+l\left(k^{2} \gamma-Y(k)\right) \lambda \sinh \left(h_{0} l\right)\right]}{k\left(k^{2}+l^{2}\right)\left(\lambda^{3}-1\right) G\left[\sinh \left[h_{0}(l+k)\right](l-k)+\sinh \left[h_{0}(l-k)\right](l+k)\right]}, \\
B_{2, s} & =h \frac{\left(l^{2}-k^{2}\right)\left[k\left(\lambda^{3}-1\right) G \cosh \left(h_{0} k\right)+\left(k^{2} \gamma-Y(k)\right) \lambda \sinh \left(h_{0} k\right)\right]}{k l\left(\lambda^{3}-1\right) G\left[\sinh \left[h_{0}(l+k)\right](l-k)+\sinh \left[h_{0}(l-k)\right](l+k)\right]} .
\end{aligned}
$$



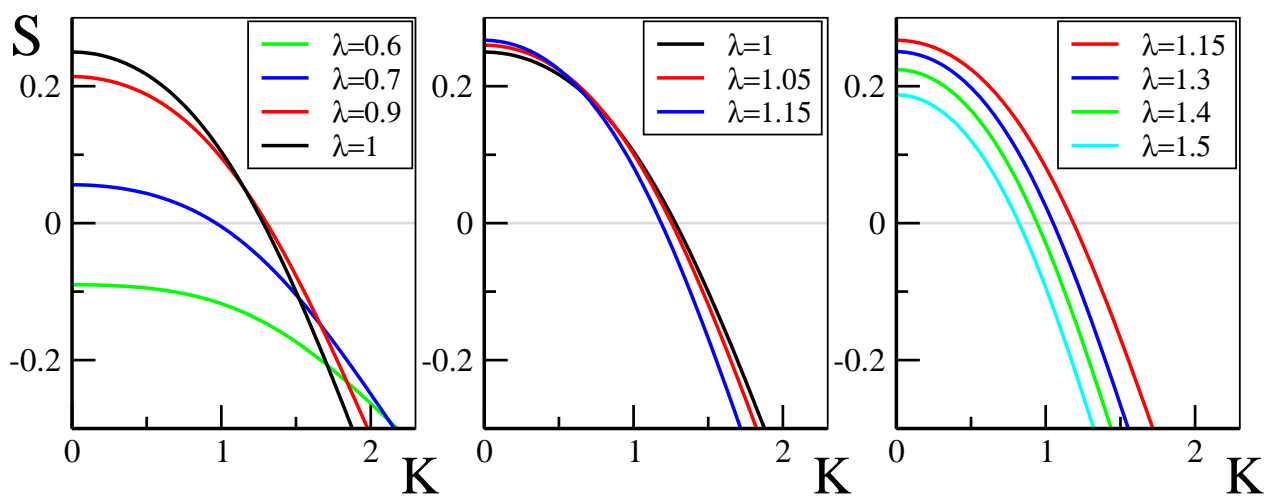

Figure 3. (Color online) The dimensionless growth rate of surface undulations, $S$, as a function of the renormalized wavenumber $K$ for the slip BC and in case of a contactor. The left panel shows increasing compression. For increasing tension, at first the growth rates slightly increase (middle panel), while for higher tension $\left(\lambda>\lambda_{s}^{*}=\left(\frac{3}{2}\right)^{1 / 3} \simeq 1.145\right)$ they again decrease (right). Parameters: $Y=5, \Gamma=0.5$.

Analogously to the previous case, the condition to have undulated solutions, Eq.(4.4), leads to an equation of the form $h Z_{s}(k)=0$ with

$$
Z_{s}=\left(\Gamma K^{2}-Y\right) \lambda\left(L^{2}-K^{2}\right)^{2}+\left(\lambda^{3}-1\right) K\left[\left(K^{2}+L^{2}\right)^{2} \operatorname{coth}(K)-4 K^{3} L \operatorname{coth}(L)\right] .
$$

By solving numerically $Z_{s}(S, K, \lambda, Y, \Gamma)=0$, one obtains the growth rates $S_{s}(K)$ as shown in Fig. 3 for the contactor and in Fig. 4 for the capacitor geometry. In the unstretched limit $\lambda \rightarrow 1$, one again gets a simple expression (shown in black in the figures),

$$
S_{s}(K, \lambda=1)=\frac{\left(Y-\Gamma K^{2}\right) \sinh (K)^{2}}{K[2 K+\sinh (2 K)]}-1 .
$$

Let us first discuss the simpler case of the contactor-induced instability. In this case, shown in Fig. 3, as the growth rate is monotonous with negative curvature, one gets the instability threshold from the limit of small wavenumbers [28]. From the growth rate $S_{s}(K, \lambda=1)=\frac{Y}{4}-1-\frac{1}{4} \Gamma K^{2}+\mathcal{O}\left(K^{3}\right)$. one immediately gets $Y_{c}=4$. Note that, in contrast to the fixed BC case, $Y_{c}$ is independent of surface tension. Considering small prestress, i.e. $\lambda=1+\delta$ with $|\delta| \ll 1$, one obtains

$$
S_{s}(K)=\frac{Y+\delta}{4}-1-\frac{1}{4}(\Gamma+\delta) K^{2}+\mathcal{O}\left(K^{3}\right) .
$$

Hence small compression $(\delta<0)$ stabilizes the system (as it increases $Y_{c}$ ), while small tension destabilizes, in agreement with Fig. 3. Note that with increasing tension, the behavior is nonmonotonous: at first the growth rates slightly increase (middle panel of Fig. 3), while for higher tensions $\left(\lambda>\lambda_{s}^{*} \simeq 1.145\right.$ ) they again decrease, see the right panel of Fig. 3. Although we can not give a closed formula for $S(K)$ for finite $\lambda$, this behavior can be explained analytically as follows: First, note that at threshold, $S\left(Y_{c}, K_{c}\right)=0$, and hence the viscosity does not play a role - the limit of instability for a viscoelastic solid is the same as for a purely elastic solid (this remark is general and independent of the BCs or the destabilization forces). Second, as the maximum of $S$ is at $K \rightarrow 0$, one can expand Eq. (4.8) with $L=K \lambda^{3 / 2}$ and $S=0$ in powers of $K$. Taking the limit $K \rightarrow 0$ one gets a condition on $Y$ leading to

$$
Y_{c}=\frac{\lambda^{3}+3}{\lambda}
$$




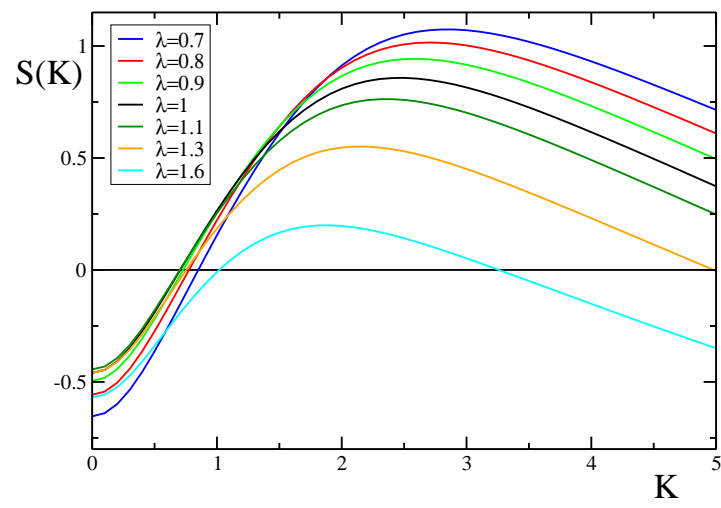

Figure 4. (Color online) The dimensionless growth rate $S$ as a function of the renormalized wavenumber $K$ for the slip BC and with an applied electric field. Both the growth rate and $K_{f g}$ increase as the compression increases ( $\lambda$ decreases). Parameters: $V=400 V, \Gamma=0.5$.
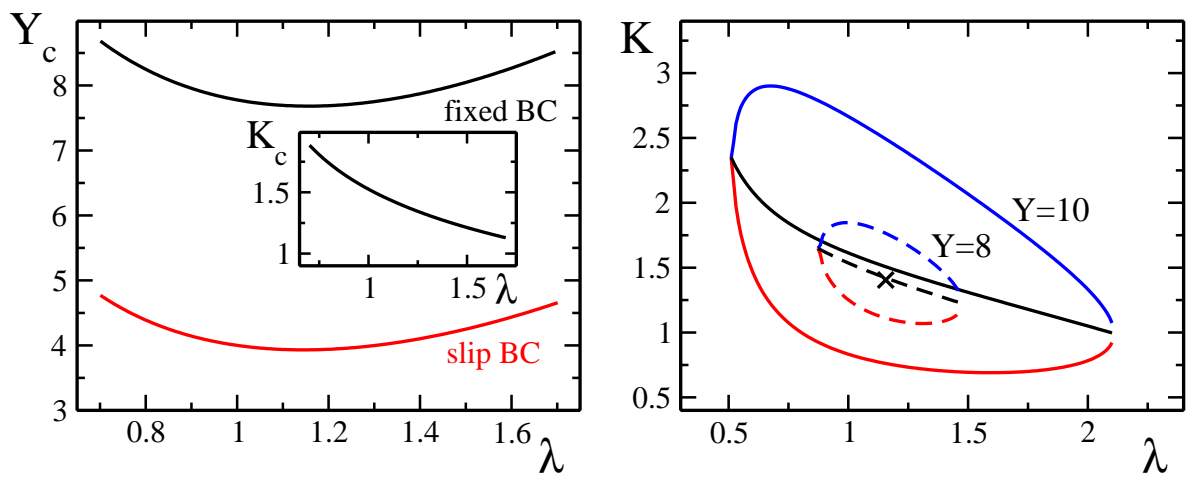

Figure 5. (Color online) Comparison of the two BCs at the substrate, for the case of the contactor-induced instability. Left panel: The dimensionless critical $Y_{c}$ as a function of stretch $\lambda$ for the fixed (black curve) and the slip BC (red). The insert represents the dimensionless critical wavenumber $K_{c}$, i.e. the wavenumber that first becomes unstable, as a function of $\lambda$ for the fixed $\mathrm{BC}$; note that for the slip BC, $K_{c}=0$. Right panel: typical wavenumbers as a function of $\lambda$ for the fixed BC: the fastest growing (black), the minimum and the maximum unstable wavenumbers (red and blue, respectively). For given stretch $\lambda$, wavenumbers between the red and blue curves will grow. $Y=10$ (solid curves), $Y=8$ (dashed), the cross marks the position of $K_{c} \simeq 1.40$, where $Y_{c, \text { min }} \simeq 7.68$ and $\lambda \simeq 1.156$. Parameter: $\Gamma=0.5$.

The red curve on the left panel of Fig. 5 is the numerically obtained $Y_{c}$ which coincides exactly with this analytical formula and displays a minimum at $\lambda_{s}^{*}=\left(\frac{3}{2}\right)^{1 / 3}$. A similar analysis is much more involved for the fixed $\mathrm{BC}$, because the selected wavenumber $K$ is finite. However, the black curve in Fig. 5 shows the numerically obtained threshold and the behavior is very similar to the case of the slip BC, albeit $Y_{c}$ is higher. Fig. 5 is further discussed when the two different BCs are compared, see below.

For the case of an electric field applied normal to the free surface, the growth rates are shown in Fig. 4. They are not monotonous anymore, showing a maximum for a certain fastest growing wavenumber $K_{f g}$. 


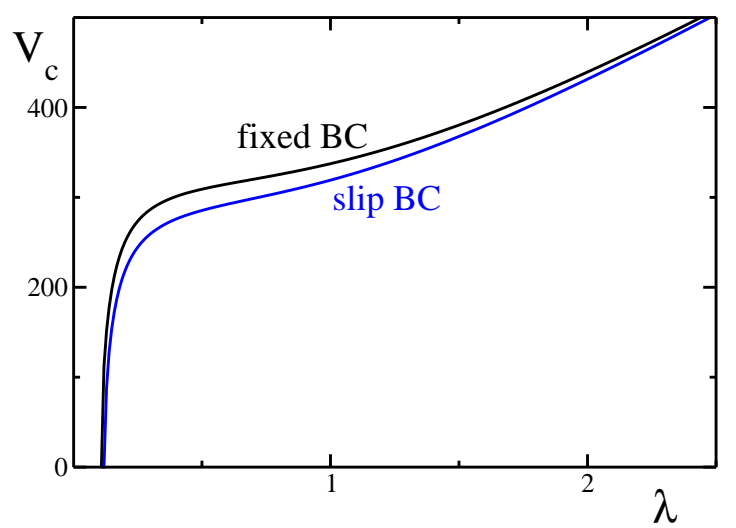

Figure 6. (Color online) The critical voltage $V_{c}$ in the capacitor geometry, as a function of the stretch factor $\lambda$ and for the fixed (black curve) and the slip BC (blue). For an important compression $\left(\lambda_{f}<0.109\right.$ and $\lambda_{s}<0.121$, however beyond the validity of the Neo-Hookean model), buckling-like undulations appear even without an electric field. Both curves show that $V_{c}$ increases when $\lambda$ increases: a stretch stabilizes the system, while compression destabilizes. The behavior of $V_{c}$ is quite similar for both BCs; Overall, with the slip BC the system is slighlty more unstable. Parameter: $\Gamma=0.5$.

In the limit $K \rightarrow 0$, we still get

$$
S_{s}(K=0)=\frac{Y(K=0)}{4}-\frac{\lambda^{3}+3}{4 \lambda},
$$

i.e. as for the conductor case. For usually applied voltages, however, small wavenumbers are damped. The growth rate increases for decreasing stretch factor $\lambda$, quite similar as in the electric destabilization with the fixed BC. Under an electric field, the compression destabilizes the system, while the stretching stabilizes the system. Numerically, we can also obtain the critical electric potential $V_{c}$ and the critical wavenumber $K_{c}$ which are, respectively, the minimum electric potential needed in order to destabilize the free surface and the wavenumber of the undulations at this critical electric potential (see Fig. 6 and the discussion below).

Discussion: We have established that for both BCs, fixed and slip, the flat film can become unstable for high enough applied voltage or van der Waals force. However, (i) the nature of the instability depends severely on the BC and (ii) the characteristic wavenumber and time scale depends on the stretch.

Now we would like to put our results in the framework of systems recently studied and discuss some of their implications. For the fixed BC, the results should be compared to those obtained in Ref. [20], which we generalized here to include prestress. The influence of the slip BC should be discussed, which corresponds to a different experimental preparation of the film (film on a incompatible brush, cf. [18], in contrast to rigidly attached film). Finally, the extension to viscoelasticity should be discussed in comparison to the purely elastic case recently studied in Ref. [6], where the stress-induced undulations develop due to surface diffusion of polymers inside the film, rather than due to viscous (or viscoelastic) flow.

Let us first discuss the effect of compression vs. tension. As already discussed above, in case of $\lambda=1$ (no stretch) we regain the results of Ref. [20]. In the case of the contactor-induced instability, the left panel of Fig. 5 shows that, for both BCs, the system is most unstable for a finite tension $\lambda_{f}^{*} \simeq 1.156$ and $\lambda_{s}^{*}=\left(\frac{3}{2}\right)^{1 / 3} \simeq 1.145$ (for the fixed and slip BC, respectively). If an electric field is used, for both BCs, the more important is the compression, the faster is the instability, while tension stabilizes the system. The nature of the destabilizing force (electric or van der Waals) modifies considerably the behavior of the 

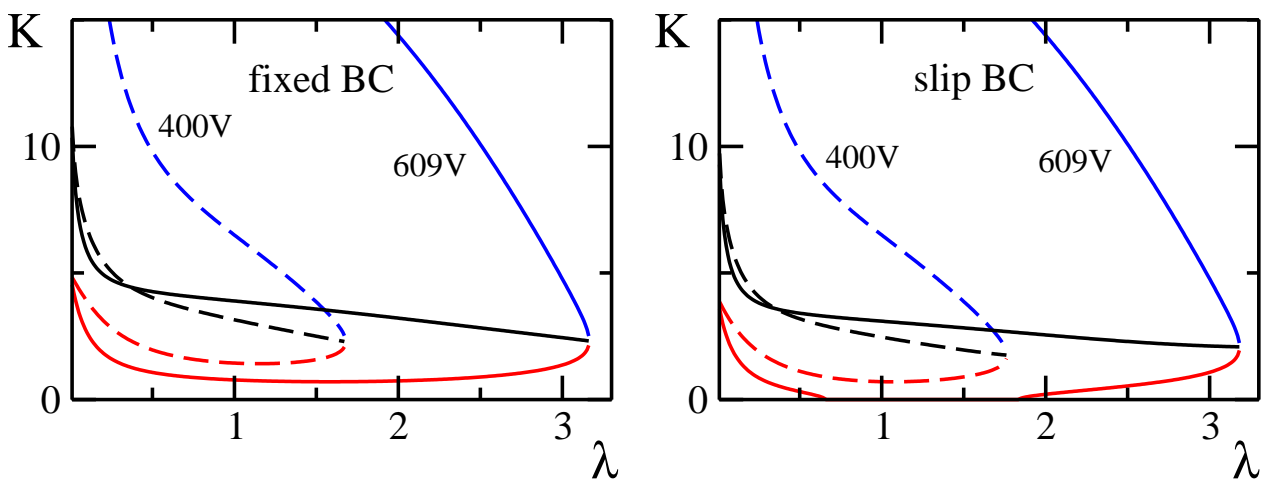

FiguRE 7. (Color online) The characteristic wavenumbers in the electrically destabilized system as a function of the stretch factor $\lambda$. Shown are the fastest growing (black) and the minimum and maximum unstable wavenumbers (red and blue, respectively). Left panel: case of the fixed BC. Right panel: case of the slip BC. For high enough values of the electric potential (cf. right panel around $\lambda=1-1.5$ ), the minimum wavenumber tends to zero, in accordance to Eq. (4.12) predicting a zero-wavenumber instability, similar to the contactor case. Parameter: $\Gamma=0.5$.

instability: the system under an electric field is stabilized by a stretch and destabilized by a compression, while the contactor system is more unstable for a finite tension. Overall, the voltage/contactor force necessary to destabilize is higher in case of the fixed BC than for the slip BC, see Figs. 5 (left panel) and 6. This is due to the fixed $\mathrm{BC}$ being much more restrictive, especially for thin films, for the displacement field inside the film. Inspecting Figs. 2 and 4, one can see that compression stabilizes (tension destabilizes) small wavenumbers while for high wavenumbers the inverse is true, i.e. $S(K)$ is higher for compression than for tension. The same has been found in Ref. [6] for the elastostatic case (i.e. without viscoelasticity), so we can conclude that viscoelasticity does not change this general trend [29]. The same trend at low wavenumbers (and not too high tension) is found for the slip BC in the contactor case, cf. Fig. 3. This means that the trend is also not sensitive to the BCs. Hence it is a purely elastic effect, associated to the Neo-Hookean model. To elucidate this point, we rederived the threshold $Y_{c}$ by using the normal stress balance, Eq. (2.14), as the solvability condition, allowing us to make connection to the the normal stress. For the slip $\mathrm{BC}$ and in leading order in $k$ we can identify

$$
\sigma_{z z} n_{z}=\frac{\lambda^{3}+3}{\lambda} h=Y h,
$$

which obviously leads to the same threshold dependence as Eq. (4.11). Hence, whithin the Neo-Hookean model, the in-plane nonlinear stretch $\lambda$ causes the asymmetric (compression vs. extension) and nonmonotonous (concerning tension) dependence of the normal stress.

For the contactor case, the main difference between the two studied BCs is that for the fixed BC one has a pattern forming instability, i.e. a finite range of wavenumbers around a certain $K_{c} \neq 0$ is growing while long and short wavenumbers are damped, cf. also the inset of the left panel of Fig. 5. $K_{c}$ is determined by $S\left(K_{c}\right)=0$ and $\frac{\partial}{\partial K} S(K)_{\mid K_{c}}=0$. Fig. 5 displays that both $K_{c}$ and $K_{f g}$ are monotonously decreasing for increasing $\lambda$. For the slip BC case, on the contrary, only the long wavelengths are unstable. In an infinite system this corresponds to $K_{c} \rightarrow 0$, while in a realistic finite system, the longest possible wavelength will grow fastest. A second important difference is that for the slip BC the critical electric field $Y_{c}$ does not depend on the surface tension.

With a destabilizing electric field, there are no such crucial differences between the two BCs. The growth rate $S(K)$ is slightly higher for the slip BC than for the fixed one. However, as observed in Fig. 7, for the slip BC, the minimum unstable wavenumber can become zero (and the system is hence 
also unstable for long wavelengths, as in the contactor case), while this is not possible for the fixed BC. This can be inferred from the limits of the growth rates at small wavenumbers: for $K \rightarrow 0$, we get $S_{f}(K=0)=-1 / \lambda<0$ but $S_{s}(K=0)=\frac{Y_{c}(K=0)}{4}-\frac{\lambda^{3}+3}{4 \lambda}$ which can become positive, depending on the value of $Y_{C}$ and the stretch factor $\lambda$.

The qualitative difference of the two instabilities (fixed vs. slip) in the contactor case can be understood as follows: in the slip BC case, the system prefers to slip (with viscous dissipation inside the film, but without losses due to friction at the substrate, as this is not yet accounted for in our model) rather than to undulate since undulations increase the surface energy while slipping can relax (elastic) energy. This explains why the selected wavenumber is $K_{c} \rightarrow 0$, in order to minimize costly undulations. The inclusion of a realistic friction mechanism with the substrate (which, however, can be nonlinear on the considered polymer-treated surfaces $[5,24,26]$ ) would be necessary to determine whether a specific wavenumber will be selected with the slip BC.

Finally, the main difference with the purely elastic case, including surface diffusion as the mechanism of material transport [6], is that the system studied here displays a threshold for the electric field. This is due to the fact that the system has to overcome the elastic restoring forces. This is best seen in the limit $\lambda \rightarrow 1, Y=0, K \rightarrow 0$, where for both BCs one has $S=-1$ (see Eqs. (4.7) and (4.12)), equivalent to $\eta s=-G$. For the Grinfeld-like mechanism studied in Ref. [6], in contrast, any variation of the elastic energy induces a gradient of the chemical potential at the surface and hence drives surface diffusion thus this system does not display a threshold. For a detailed discussion of the dependence of the elastic instability on the stretch we refer to our previous work Ref. [6].

\section{Conclusions}

This work has been motivated by experiments studying the electro-hydrodynamic or contactor-induced instability of spin-cast, and hence presumably stressed, thin polymer films. We have investigated theoretically the effect of in-plane stress on the stability of a viscoelastic thin polymer film in a normal electric field, or in presence of a closeby contactor inducing van der Waals forces. We find that the residual stress affects both the growth rates (i.e. the typical timescale of the instability) and the unstable wavelengths. Moreover, with a contactor the system is highly sensitive to the boundary conditions holding at the film-substrate interface, as demonstrated here for two distinct cases of a rigidly attached film (where a finite wavelength instability occurs) and a film allowing for slip at the substrate (where a long wavelength instability occurs). In the electrically destabilzed case, the differences are only quantitative.

We studied here only the simpler case of a viscoelastic solid (Voigt-Kelvin model). It would be interesting, and more realistic in view of polymer films liquefied by heating above the glass transition, to also study the case of a viscoelastic fluid (Maxwell model). Furthermore, in case of the BC with slip it would be interesting to include the friction with the substrate into the description. Then a new length scale, the so-called slip length, naturally emerges which may introduce a characteristic wavelength in the corresponding instability (while so far $K_{c}=0$ in the slip case).

Acknowledgements. F. Z. thanks the DFG for funding via IRTG 1642 Soft Matter Science.

\section{References}

[1] M. Adda-Bedia, L. Mahadevan. Crack-front instability in a confined elastic film. Proc. R. Soc. Lond. A, 462 (2006) 3233-3251.

[2] R. J. Asaro, W. A. Tiller. Interface morphology development during stress corrosion cracking: Part I. Via surface diffusion. Metall. Trans. B 3 (1972), 1789-1796.

[3] D. R. Barbero, U. Steiner. Nonequilibrium Polymer Rheology in Spin-Cast Films. Phys. Rev. Lett. 102 (2009), 248303, $1-4$.

[4] H. Bodiguel, C. Fretigny. Viscoelastic dewetting of a polymer film on a liquid substrate. Eur. Phys. J. E, 19 (2006), 185-193.

[5] L. Bureau, L. Léger. Sliding Friction at a Rubber/Brush Interface. Langmuir 20 (2004), 4523-4529. 
[6] F. Closa, F. Ziebert, E. Raphaël. Interplay of internal stresses, electric stresses, and surface diffusion in polymer films. Phys. Rev. E, 83 (2011), 051603, 1-13.

[7] A. Ghatak. Confinement-induced instability of thin elastic film. Phys. Rev. E, 73 (2006), 041601, 1-7.

[8] A. Ghatak, M. K. Chaudhury. Critical Confinement and Elastic Instability in Thin Solid Films. J. Adh., 83 (2007), 679-704.

[9] A. Ghatak, M. K. Chaudhury, V. Shenoy, A. Sharma. Meniscus instability in a thin elastic film. Phys. Rev. Lett., 85 (2000), 4329-4332.

[10] M. A. Grinfeld. Instability of the interface between a nonhydrostatically stressed elastic body and a melt. Dok. Akad. Nauk SSSR 290 (1986), 1358-1363 [Sov. Phys. Dokl. 31 (1986), 831-834].

[11] J. D. Jackson. Classical Electrodynamics. Wiley, New York (1998).

[12] L. D. Landau, E. M. Lifshitz. Theory of Elasticity. Pergamon Press, New York, 1986.

[13] J. R. Melcher. Electrohydrodynamic and Magnetohydrodynamic Surface Waves and Instabilities . Phys. Fluids, 4 (1961), 1348-1354.

[14] C. W. Macosko. Rheology: Principles, Measurements, and Applications. Wiley, New York (1994).

[15] K. Norrman, A. Ghanbari-Siahkali, N. B. Larsen. Studies of spin-coated polymer films. Annu. Rep. Prog. Chem., Sect. C, 101 (2005), 174-201.

[16] A. Onuki. Interface instability induced by an electric field in fluids. Physica A 217 (1995), 38-52.

[17] K. R. Rajagopal, G. Saccomandi. Shear Waves in a Class of Nonlinear Viscoelastic Solids. Q. J. Mechanics Appl. Math. 56 (2003), 311-326.

[18] G. Reiter, M. Hamieh, P. Damman, S. Sclavons, S. Gabriele, T. Vilmin, E. Raphaël. Residual stresses in thin polymer films cause rupture and dominate early stages of dewetting. Nature Mat., 4 (2005), 754-758.

[19] E. Schäffer, T. Thurn-Albrecht, T. P. Russell, U. Steiner. Electrohydrodynamic instabilities in polymer films. Europhys. Lett. 53 (2001), 518-524.

[20] V. Shenoy, A. Sharma. Stability of a thin elastic film interacting with a contactor. J. Mech. Phys. Solids 50 (2002), 1155-1173.

[21] G. I. Taylor, A. D. McEwan. The stability of a horizontal fluid interface in a vertical electric field. J. Fluid Mech., 22 (1965), 1-15.

[22] G. Tomar, V. Shankar, A. Sharma, G. Biswas. Electrohydrodynamic instability of a confined viscoelastic liquid film. J. Non-Newtonian Fluid Mech. 143 (2007), 120-130.

[23] Y. Tsori. Phase transitions in polymers and liquids in electric fields. Rev. Mod. Phys. 81 (2009), 1471-1494.

[24] T. Vilmin, E. Raphaël, P. Damman, S. Sclavons, S. Gabriele, M. Hamieh, G. Reiter. The role of nonlinear friction in the dewetting of thin polymer films. Europhys. Lett. 73 (2006), 906-912.

[25] T. Vilmin, F. Ziebert, E. Raphaël. Simple View on Fingering Instability of Debonding Soft Elastic Adhesives. Langmuir, 26 (2010), 3257-3260.

[26] F. Ziebert, E. Raphaël. Dewetting of thin polymer films: Influence of interface evolution. EPL 86 (2009), 46001, p1-p6.

[27] F. Ziebert, E. Raphaël. Dewetting dynamics of stressed viscoelastic thin polymer films. Phys. Rev. E, 79 (2009), 031605, $1-10$.

[28] F. Ziebert, W. Zimmermann. Comment on "Instabilities of Isotropic Solutions of Active Polar Filaments". Phys. Rev. Lett. 93 (2004), 159801-1.

[29] Note that $Y_{c}, K_{c}$ and $K_{f g}$ are determined by elasticity, the viscosity does not play a role. However, $K_{f g}$ can exist only if $\eta \neq 0$, else, the problem is a static problem and $K_{f g}$ is not defined. 